\title{
Técnicas para aumentar la Seguridad de la Operación en Condiciones de Red Degradada a través de Modificaciones Topológicas en Sistemas de Potencia
}

\author{
Carlos M. Quintero, Jesús M. López-Lezama y Nicolás Muñoz-Galeano \\ Grupo de Investigación GIMEL, Departamento de Ingeniería Eléctrica, Facultad de Ingeniería, Universidad de Antioquia, \\ Calle 67 No.53-108, Medellín - Colombia (e-mail: manuel.quintero@udea.edu.co; jmaria.lopez@udea.edu.co; \\ nicolas.munoz@udea.edu.co).
}

Recibido May.7, 2019; Aceptado Jul. 3, 2019; Versión final Ago. 20, 2019, Publicado Dic. 2019

\begin{abstract}
Resumen
En este trabajo se proponen dos técnicas metaheurísticas de identificación de escenarios topológicos de redes de transmisión para aumento de seguridad en condiciones de red degradada. Las técnicas implementadas son el recocido simulado (Simulated Annealing) y la búsqueda local iterada (Iterated Local Search). El objetivo de las modificaciones topológicas consiste en disminuir el número de restricciones violadas ante contingencias $\mathrm{N}-1$. Las técnicas de búsqueda propuestas en este trabajo realizan exploraciones topológicas a través del cambio de estado de los interruptores de las subestaciones. Estas encuentran reconfiguraciones del sistema eléctrico de potencia que garantizan un nivel de seguridad mayor ante contingencias $\mathrm{N}-1$ que el obtenido en el caso sin cambios en la topología. Las condiciones de seguridad consideran un modelo $\mathrm{AC}$ de la red y un modelo bahía-interruptor para la evaluación de contingencias, lo que permite la evaluación de las condiciones reales de liberación de fallas en el sistema de potencia. Se presentan resultados sobre el sistema IEEE de 118 barras que evidencian la efectividad y aplicabilidad de las técnicas propuestas.
\end{abstract}

\section{Techniques to Increase Security Operation in Degraded Network Conditions through Topological Modifications in Power Systems}

\begin{abstract}
This paper presents two techniques for identifying topological scenarios of transmission networks for security enhancement in degraded network conditions. The implemented techniques are Simulated Annealing and Iterated Local Search. The objective of the topological modifications is the reduction of the number of violated constraints under $\mathrm{N}-1$ contingencies. The proposed search techniques make topologic explorations through state changes in sub-station switches. These explorations are used to find reconfigurations of the electric power system which guaranty a higher level of security in face of $\mathrm{N}-1$ contingencies when compared with the case when the topology is not changed. Security conditions consider an AC model and a bay-switch model to the assessment of contingencies, which allows the evaluation of real conditions of failure release in the power system. Results are presented with the IEEE 118 bus test system that demonstrates the effectiveness and applicability of the proposed techniques.
\end{abstract}

Keywords: power systems; substations; security; network reconfiguration 


\section{INTRODUCCIÓN}

La operación de un sistema eléctrico de potencia (SEP) tiene como objetivo garantizar el suministro de energía eléctrica, minimizando los costos operativos y cumpliendo con ciertos niveles de calidad, confiabilidad y seguridad (Parvini et al, 2018; Yang et al, 2019). En este contexto, la optimización de los recursos es una prioridad para los operadores de red. La operación de un SEP es impactada por una serie de factores como las exigencias regulatorias, fenómenos climáticos, eventos sociopolíticos (condiciones anormales de orden público) y fallas geológicas que pueden llevar el sistema a un nivel de estrés mucho mayor. Por otro lado, el retraso en las obras de expansión y el continuo crecimiento de la demanda provocan la agudización de las restricciones, aumentando la probabilidad de entrar en escenarios de operación que no permiten cumplir con los criterios de confiabilidad y seguridad exigidos por el regulador (Saldarriaga-Zuluaga, et al, 2018). Las condiciones de orden público, así como eventos sociales y políticos de gran magnitud pueden tener un impacto negativo en la operación de los SEP. Una práctica común en la operación de los SEP es modificar los criterios de confiabilidad, buscando cubrir un mayor número de escenarios ante los cuales se pueda presentar una desatención de la demanda. Lo anterior tiene una implicación en el número de restricciones que se activan y el costo para el cubrimiento de las mismas (Jiajun et al, 2017).

En la literatura técnica se han presentado diferentes estudios que abordan la modificación topológica de los SEP con diferentes propósitos, como el alivio de sobrecargas (Mazi et al., 1986; Shao et al., 2005), mejoramiento del perfil de tensiones (Bai et al., 2016; Potluri et al., 2012; Soroush et al., 2014), aumento de la confiabilidad (Zhang et al., 2014) y disminución de los costos de operación (Fuller et al., 2012; Khanabadi, et al., 2013). En estos trabajos, el planteamiento de las soluciones se basa en la apertura de las líneas de transmisión y división de las subestaciones. La primera solución hace referencia a encontrar un enlace para ser sujeto al cambio de estado y la segunda busca hallar la mejor distribución posible en cada barra de las bahías de línea o de transformación en una subestación. Ambas soluciones tienen como objetivo mejorar las condiciones de la red eléctrica en términos de seguridad y economía, respecto las que se tenían previo a la implementación de las modificaciones. Los trabajos antes mencionados no consideran el cambio de estados de todos los interruptores asociados a las subestaciones. Otras metodologías, como las presentadas en (Granelli et al., 2006; Heidarifar et al., 2016; Korad et al., 2013) planean los cambios topológicos a partir del cambio de estado de los interruptores; sin embargo, las subestaciones que se consideran son de configuración doble barra e interruptor y medio, dejando por fuera las subestaciones tipo anillo que pueden aprovecharse para la reconfigurar el sistema y obtener beneficios en términos de seguridad.

En este trabajo se consideran dentro del modelo tres tipos de subestaciones que permiten hacer reconfiguraciones topológicas en el sistema. Estos tipos son conocidos como interruptor y medio, doble barra y anillo. Para resolver el modelo de optimización propuesto se implementaron dos técnicas de búsqueda: Recocido Simulado (SA, Simulated Annealing) y Búsqueda Local Iterada (ILS, Iterated Local Search). Estos métodos hacen una exploración de topologías en un SEP a través de la modificación de los estados de los interruptores pertenecientes a las subestaciones con configuraciones tipo interruptor y medio, doble barra y anillo. Las técnicas están soportadas por un pre procesamiento de la información que les permite acceder a los componentes necesarios del sistema en forma sistemática para realizar las modificaciones de manera eficiente, del mismo modo facilita la interpretación de los resultados. La contribución principal de este artículo, con respecto a otros trabajos reportados en la literatura especializada, radica en la búsqueda de topologías adicionales a las que se pueden hallar en subestaciones tipo doble barra, y en explorar las características que brindan las subestaciones tipo anillo e interruptor y medio, para mejorar las condiciones de seguridad en un SEP con red degradada. En este contexto, se entiende por red degradada aquella que se encuentra en un punto de operación en que no se pueden cumplir todas las condiciones de seguridad, bien sea por disponibilidad de red o disponibilidad energética. Adicionalmente, la evaluación de las condiciones de seguridad se hace considerando un modelo AC de la red y además considera un modelo bahía - interruptor para la evaluación de contingencias. Esto último permite hacer una evaluación de las condiciones reales de liberación de fallas en el sistema de potencia, ya que el análisis de contingencias utiliza los interruptores adyacentes al punto de falla para su despeje.

\section{DEFINICIÓN DEL PROBLEMA}

En (1) se presenta la función objetivo que califica una topología de acuerdo a la cantidad de contingencias que sea capaz de soportar sin ocasionar violaciones de seguridad, considerando que la demanda y generación deben permanecer constantes. El primer término representa el número de sobrecargas ante una contingencia. En este caso, $O L_{i, l}$ es una variable binaria que toma el valor de uno si la contingencia $i$ produce una sobrecarga en el elemento / y cero en caso contrario. El segundo término representa el número de violaciones de tensión de las barras ante una contingencia. En este término la variable binaria $O V_{i, n}$ toma 
el valor de uno si la contingencia $i$ produce una tensión por fuera de los límites en la barra $n$. El tercer término es una variable binaria que activa una penalización a la función objetivo ante la no convergencia del flujo de carga por una contingencia. La variable binaria $C V_{r}$ toma el valor de uno cuando no hay convergencia ante la contingencia $r$. El cuarto término de la función objetivo representa el número de generadores o cargas que fueron desconectadas como producto de la reconfiguración topológica, la variable binaria $D X_{s}$ toma el valor de uno cuando se considera una carga o generador $s$ es desconectado y cero en caso contrario. Los últimos dos términos se multiplican por los factores de penalización $k_{1}$ y $k_{2}$.

Minfo $=\sum_{i \in C e} \sum_{l \in B r} O L_{i, l}+\sum_{i \in C e} \sum_{n \in N} O V_{i, n}+k_{1} * \sum_{r \in N C e} C V_{r}+k_{2} * \sum_{s \in D c g} D X_{S}$

En todas las variaciones topológicas del sistema se debe garantizar que se cumplan las restricciones de balance de potencia activa y reactiva. Por otro lado, se debe mantener la generación constante, esto está dado por las ecuaciones (2) a (10). Ambos tipos de restricciones son consideradas de forma implícita mediante el software de análisis de SEP. La restricción (2) indica que la demanda no atendida debido a la desconexión de cargas debe ser menor a la demanda del nodo. Las restricciones (3) y (4) indican las expresiones matemáticas de las inyecciones de potencia nodal. La ecuación (5) indica los componentes de potencia activa y reactiva presentes en la potencia aparente de las ramas del sistema. Las ecuaciones (6) y (7) son las expresiones matemáticas de la potencia activa y reactiva en las ramas del sistema, respectivamente. Las ecuaciones (8) y (9) indican las restricciones de balance de potencia activa y reactiva por nodo, respectivamente. Finalmente, la restricción (10) indica que el ángulo de referencia debe ser cero.

$0 \leq\left(P_{D S_{n}}\right) \leq P_{D_{n}}, \quad \forall n \in N$

$P_{n}=V_{n} \sum_{n} V_{m}\left[G_{m n} \cos \left(\theta_{m n}\right)+B_{m n} \sin \left(\theta_{m n}\right)\right], \quad \forall l \in B r, \quad \forall n \in N$

$Q_{n}=V_{n} \sum_{n} V_{m}\left[G_{m n} \sin \left(\theta_{m n}\right)-B_{m n} \cos \left(\theta_{m n}\right)\right], \quad \forall l \in B r, \quad \forall n \in N$

$\left(S_{l}^{B r}\right)^{2}=\left(P_{l}^{B r}\right)^{2}+\left(Q_{l}^{B r}\right)^{2}, \quad \forall l \in B r$

$P_{l}^{B r}=g_{m n} V_{n}^{2}+g_{m n} V_{m} V_{n} \cos \left(\theta_{m n}\right)-b_{m n} V_{m} V_{n} \sin \left(\theta_{m n}\right), \quad \forall l \in B r$

$Q_{l}^{B r}=-b_{m n} V_{n}^{2}+g_{m n} V_{m} V_{n} \cos \left(\theta_{m n}\right)-b_{m n} V_{m} V_{n} \sin \left(\theta_{m n}\right), \quad \forall l \in B r$

$P_{g}^{G e n}-P_{D_{n}}+P_{D S_{n}}=P_{n}, \quad \forall n \in N$

$Q_{g}^{G e n}-Q_{D_{n}}+Q_{D S_{n}}=Q_{n}, \quad \forall n \in N$

$\theta_{\text {ref }}=0$

\section{TÉCNICAS DE SOLUCIÓN}

Previo a la aplicación de las técnicas de búsqueda topológica, se realiza un pre proceso que tiene como objetivo almacenar de forma estructurada la información topológica de interés. El almacenamiento estructurado de la información reduce el esfuerzo computacional para encontrar y acceder los componentes del SEP que se van a modificar; además, evita evaluar condiciones topológicas eléctricamente equivalentes. En la Fig. 1 se ilustra el proceso de pre procesamiento. El almacenamiento estructurado de la información se realiza a través de vectores como se muestra en la

Fig. 2. El orden en el que se realiza la caracterización de estados es de acuerdo al tipo de subestación, comenzado por las tipo interruptor y medio para continuar con las doble barra y anillo, respectivamente. Como no es práctico asignar todos los elementos del diámetro en una posición, se toma como referencia el elemento conectado a barra 1. En caso de que este elemento no exista para el diámetro se tomará el elemento 2 perteneciente al mismo diámetro. En las subestaciones tipo doble barra y anillo la asignación correspondiente a la posición de las bahías se realiza a través del elemento asociado a la misma. 
SA e ILS son técnicas de búsqueda basados en una solución; lo que significa que toda la exploración se realiza buscando mejorar un candidato de solución y no un conjunto de soluciones. Estos métodos están compuestos normalmente por dos etapas. En la primera se construye la solución inicial y en la segunda se mejora esta. La solución inicial para el problema abordado está dada por el estado actual del sistema; es decir, con los estados los interruptores y seccionadores que se opera en condiciones normales (recomendados por los equipos de planeación operativa). Sin embargo, las técnicas de búsqueda pueden operar en caso de cambiar la solución inicial. Esta característica permite inicializar los métodos de búsqueda desde puntos de operación que se tienen identificados como capaces de mejorar las condiciones de seguridad; de esta manera, se puede aprovechar la experiencia operativa sobre el SEP.

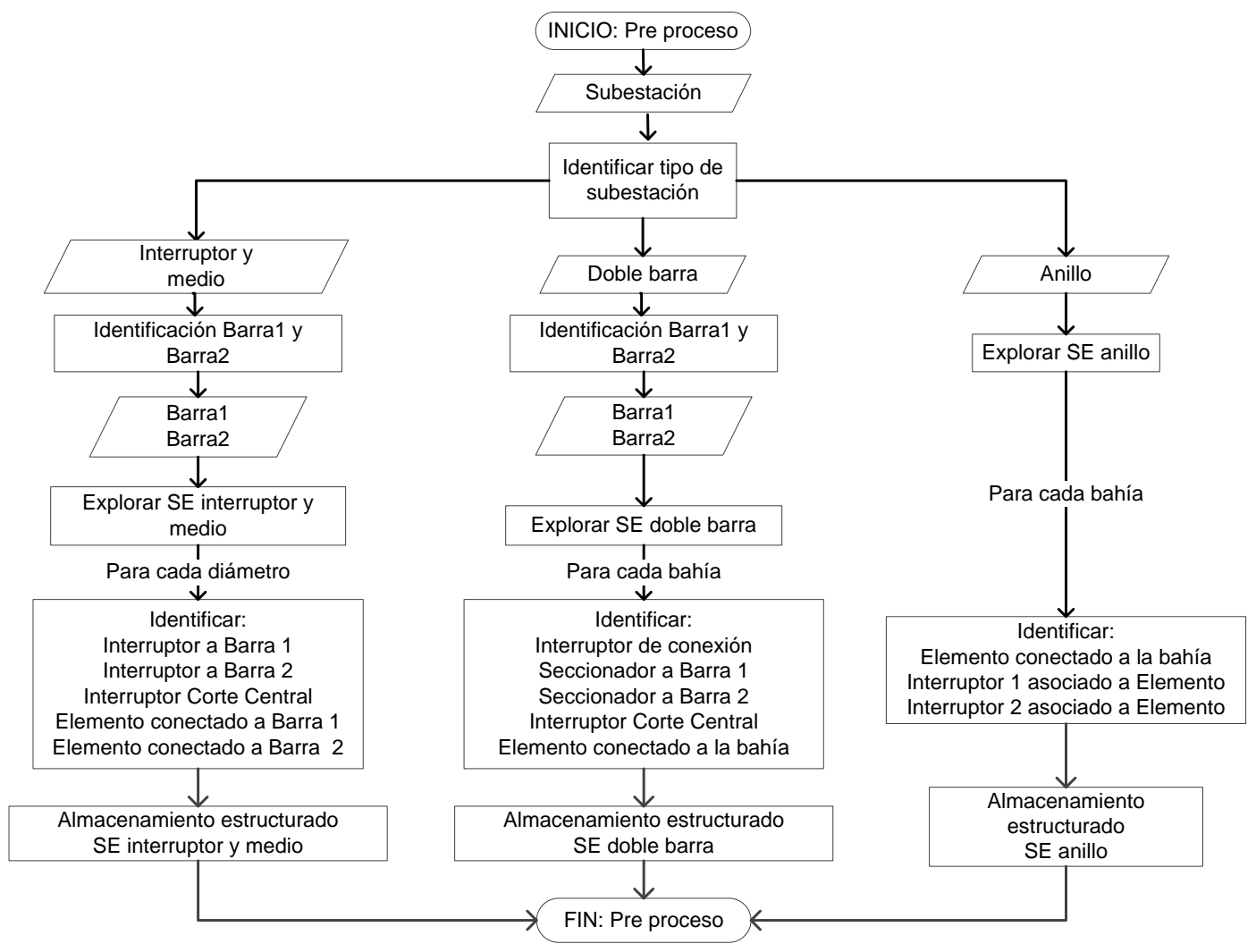

Fig. 1: Pre proceso de la información asociada a las subestaciones.

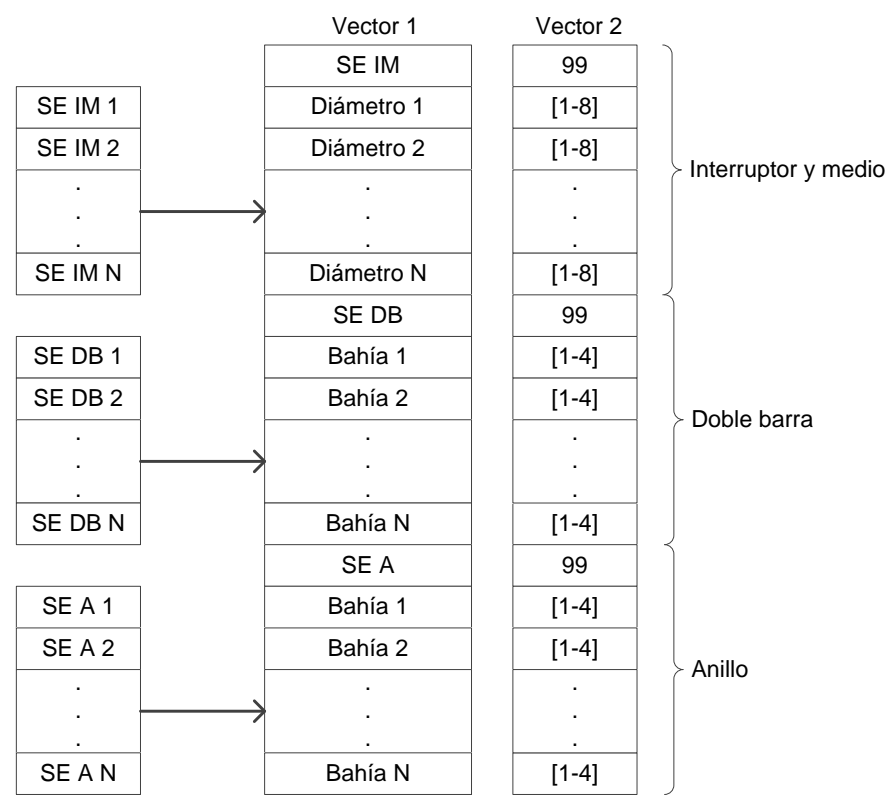

Fig. 2: Solución en representación vectorial. 


\section{Recocido Simulado (SA)}

El Recocido Simulado es una variante de la búsqueda local que permite movimientos ascendentes para evitar quedar atrapado prematuramente en un óptimo local. Esta metaheurística ha demostrado ser una herramienta exitosa para resolver una amplia gama de problemas de optimización como se reporta en (López et al., 2015; Rodriguez et al., 2016). El SA implementado se ilustra en la Fig. 3. En la primera etapa se selecciona una solución inicial (So) y se asignan valores a los parámetros del algoritmo. La solución inicial consiste en un punto de operación caracterizado vectorialmente por el estado de los interruptores (ver Fig. 2). Los parámetros del algoritmo son la temperatura inicial $\left(\mathrm{T}_{0}\right)$, el valor inicial de la variable de control (T), la temperatura final (Tfin) y número de iteraciones (nrep). El algoritmo utiliza una función de reducción de temperatura encargada de determinar el enfriamiento del sistema. En este caso, se utilizó una función de enfriamiento lineal, con una constante de reducción $d t$.

En la etapa de enfriamiento el SA realiza exploraciones topológicas de acuerdo al movimiento seleccionado. Este es variable y depende del número de subestaciones que conformen la solución y el número de bahías o diámetros que tenga la subestación que fue seleccionada para realizar cambios a los estados de sus interruptores o seccionadores. El movimiento aleatorio determina cuantas y cuales subestaciones, que bahías o diámetros y los cambios de estado que se harán a interruptores y seccionadores durante la ejecución del mismo. A los interruptores y seccionadores seleccionados de forma aleatoria se le deben asignar nuevos estados de abierto o cerrado considerando el tipo de subestación al que pertenezcan. La función objetivo es calculada después de la aplicación de cada movimiento y su valor es comparado con la mejor solución encontrada hasta el momento (incumbente).

Si el valor de la solución actual es menor, esta reemplaza la incumbente actual. Cuando el valor de la solución actual es mayor al de la incumbente se genera un número aleatorio "u» entre 0 y 1 . Este número es comparado con $e^{-\beta / T}$, donde $\beta$ es la diferencia de funciones objetivo y $T$ es la temperatura del sistema en la cual se encontró la topología. Si «u» es menor que $e^{-\beta / T}$, se acepta la solución y se continua con el proceso de exploración. En caso contrario, se rechaza la nueva solución y se continúa con el algoritmo. El control que se hace sobre la aceptación o rechazo de las soluciones depende de la temperatura. Cuando se encuentra más elevada, la probabilidad de aceptación de soluciones peores se incrementa, brindándole diversidad a la búsqueda. Por otro lado, cuando la temperatura disminuye, la aceptación de soluciones de poca calidad disminuye, lo que significa que se intensifica la búsqueda alrededor de la mejor solución encontrada.

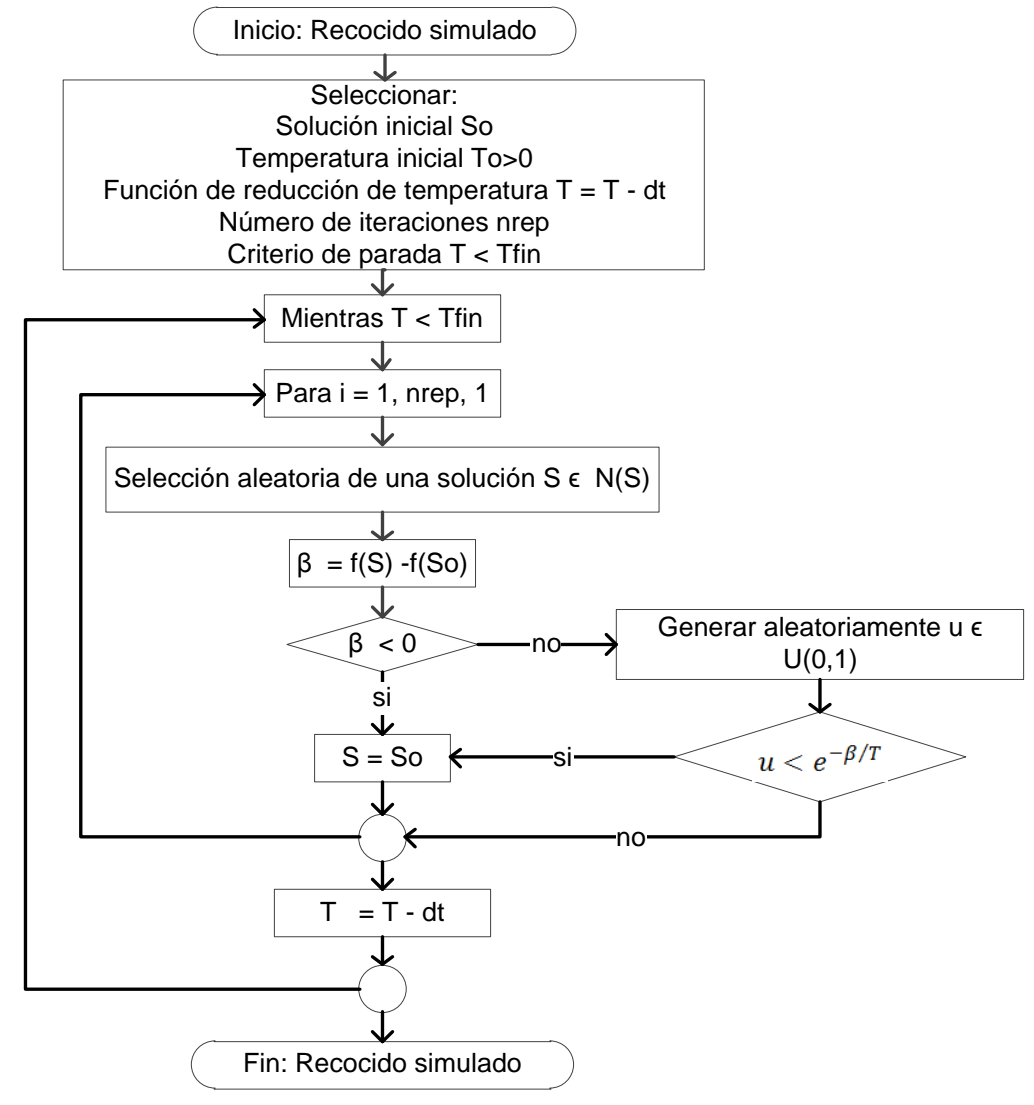

Fig. 3: Recocido Simulado aplicado a la búsqueda topológica. 


\section{Búsqueda Local Iterada (ILS)}

La ILS consiste en aplicar iterativamente un procedimiento de búsqueda local a soluciones iniciales aleatorias. El proceso inicia con una solución aleatoria o pseudo-aleatoria, sobre la cual se hace una búsqueda local. Posteriormente, se genera una nueva solución (reinicialización) a partir del óptimo local encontrado y se realiza otra búsqueda local. El proceso se repite de forma iterativa hasta que se cumple un número de ciclos dado. Normalmente se realiza una modificación suave para la búsqueda local y una fuerte para la reinicialización. Esta técnica ha sido utilizada en diferentes aplicaciones como se indica en (López et al., 2017). En la Fig. 4 se ilustra la ILS aplicada a la búsqueda de topologías en SEP. El procedimiento es inicializado con una solución $S$. Para la inicialización de $S$ se toma como referencia el punto de operación actual del SEP con el estado de los interruptores cerrado, incluyendo los acoples de barra, donde cada subestación representada un nodo eléctrico.

Sin embargo, es posible comenzar en un punto de operación diferente cambiando el estado de los interruptores o seccionadores que se quieran modificar. La caracterización de la solución para la ILS es vectorial como se muestra en la

Fig. 2. Los parámetros utilizados cumplen las siguientes tareas dentro del algoritmo de búsqueda aplicado. El número de iteraciones (nrep) determina la cantidad de veces que se ejecutará el procedimiento de búsqueda local más reinicialización. La distancia del movimiento $k$ establece el número de bahías o diámetros que serán intervenidos en cada subestación perteneciente a la solución. El número de ciclos establece el número de intentos que se tienen para encontrar una solución diferente a la actual y que cumpla con el criterio de factibilidad que establece una máxima distancia entre la incumbente y la nueva solución.

El movimiento aplicado en la búsqueda local está definido por la constante de distancia $k$, que determina cuántas bahías o diámetros de una subestación son sujetas al cambio de estado de sus interruptores asociados. Tanto la selección de las bahías o diámetros como los cambios de estado son asignados de manera aleatoria. La búsqueda local establece que para todas las subestaciones consideradas en la solución es aplicado el movimiento y posteriormente se calcula el valor de la función objetivo con la nueva topología. Este es comparado con la incumbente. En caso de que la última solución sea mejor, esta será almacenada y reemplazará a la incumbente actual.

Después de aplicar un movimiento a una subestación y calcular la función objetivo se regresa a la solución inicial. El segundo bloque del algoritmo es la reinicialización. El objetivo de esta es encontrar un nuevo punto de inicio para la búsqueda local y hacer una exploración diferente del espacio de solución. Esto aplica diversificación a la búsqueda y permite escapar de óptimos locales. La reinicialización está formada por un bucle que tiene como variable un número de ciclos y un parámetro de distancia entre la solución encontrada en la reinicialización y la incumbente. El control de factibilidad se realiza estableciendo una distancia máxima entre la solución encontrada en la reinicialización y la solución incumbente. Solo se acepta una nueva solución sí la diferencia entre las funciones objetivo es menor a cierto número de violaciones adicionales de seguridad. De esta forma, si después de aplicar los movimientos establecidos en la reinicialización no se encuentra una solución que cumpla con la distancia requerida se repite el ciclo el número de veces establecido por el parámetro de control. Si después de agotar todos los ciclos las soluciones halladas no cumplen con el criterio de distancia respecto a la solución incumbente, se selecciona esta última para continuar con la búsqueda local.

\section{RESULTADOS Y DISCUSIÓN}

Para validar la aplicabilidad de la metodología propuesta se utilizó el sistema de prueba IEEE de 118 barras. Este sistema cuenta con 184 ramas, 91 cargas y 19 generadores. Para realizar flujos de carga y análisis de contingencias, es necesario realizar un modelado del sistema considerando las características de bahía interruptor de los componentes del SEP que se conectan a cada subestación. Esto implica que cada subestación debe estar modelada con todos los seccionadores e interruptores que componen cada una de las bahías de conexión de los diferentes elementos del SEP. En la Fig. 5 se muestra la forma en que se conectan los elementos del SEP a los diferentes tipos de configuración de subestaciones consideradas en este trabajo.

Todas las subestaciones del sistema IEEE de 118 barras son del tipo barra sencilla. Esto implica que la conexión de cada elemento del SEP a las subestaciones es a través de un solo interruptor. Para la ejecución de las pruebas fue necesario realizar una serie de transformaciones para agregar flexibilidad a las subestaciones. Para ello se desarrolló un algoritmo de transformación de tipo de configuración de subestación con capacidad de transformar una subestación tipo barra sencilla a interruptor y medio, doble 
barra o anillo (Quintero et al., 2018). El SEP con sus modificaciones fue modelado en DIgSILENT PowerFactory. Este software fue seleccionado por sus características de velocidad de cálculo, multi procesamiento, acceso a objetos y resultados, flexibilidad de implementación y por ser un software de análisis de sistemas de potencia usado por diferentes operadores de red del mundo. En cuanto a las características de procesamiento requeridas, vale la pena mencionar que el algoritmo puede ser ejecutado en cualquier equipo que soporte el software DIgSILENT PowerFactory. La velocidad de los cálculos dependerá del tamaño y complejidad del sistema bajo evaluación y la capacidad de cómputo de la máquina donde se ejecute el programa.

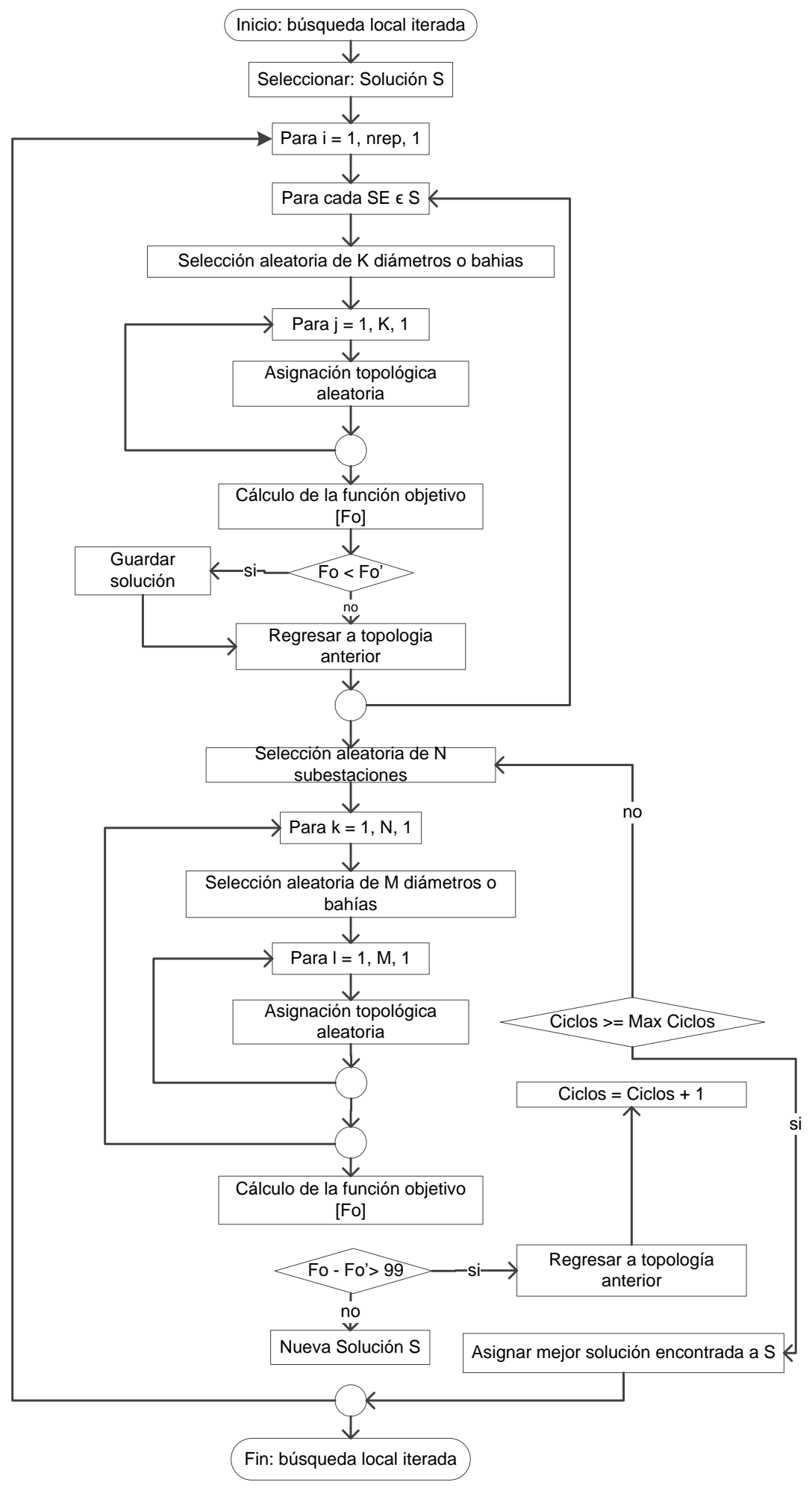

Fig. 4: Búsqueda Local Iterada aplicada a la búsqueda topológica. 
Las subestaciones de los nodos $23,47,32,42,66,45,62,51,49,69,54,50,48$ fueron exploradas para encontrar topologías que mejoran la seguridad del SEP de prueba. Las trece subestaciones consideradas en este primer grupo de simulaciones son de tipo interruptor y medio. El objetivo de este primer grupo de simulaciones es determinar los parámetros de ajuste de los métodos de búsqueda implementados. Las contingencias en el análisis de seguridad fueron de tipo N-1 para todas las ramas, exceptuando los transformadores de uso exclusivo de conexión de generadores. Esto para que tanto la generación como la demanda se mantengan constantes durante los análisis. Se resalta el hecho de que el análisis de contingencias se realiza despejando las fallas con los interruptores adyacentes al elemento fallado. Para el caso base se considera que todas las subestaciones tienen sus interruptores cerrados, lo cual es equivalente a tener por cada subestación un solo nodo eléctrico. Para el caso base, el flujo de carga converge para todas las contingencias; además, todos los elementos de generación y demanda se encuentran conectados. Por lo tanto, el valor de la función objetivo evaluada en el caso base obedecerá al resultado del término asociado a las sobrecargas. La función objetivo para el caso base es 65, que corresponde al número de violaciones a los límites de cargabilidad o tensión.
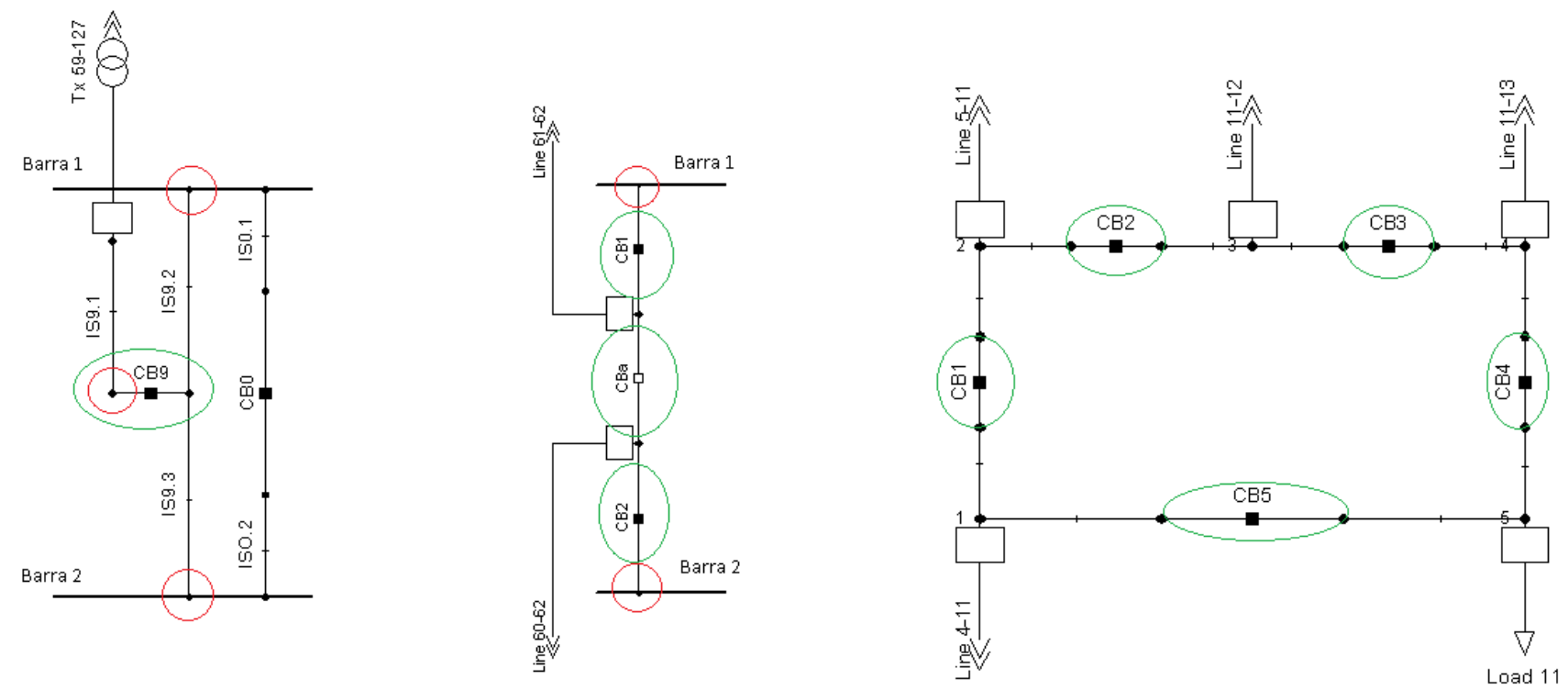

Fig. 5: Modelo bahía - interruptor para conexión a subestaciones de diferentes tipos.

\section{Solución utilizando ILS}

Con el método ILS se realizaron veinte simulaciones; combinando los parámetros de distancia, ciclos y número de iteraciones que están asociados a la diversificación, control de factibilidad y parada de la simulación, respectivamente. En la Tabla 1 se presentan los resultados obtenidos. Estos muestran que el número de ciclos empleados durante la reinicialización de la solución no genera ventajas cuando el número de iteraciones es lo suficientemente alto. Cuando el número de iteraciones es menor, la componente de diversificación que brinda el parámetro de distancia favorece la búsqueda de topologías con mejores condiciones de seguridad. Las mejores soluciones se encontraron con mayor tiempo de simulación que a su vez implica tener mayor número de iteraciones, distancia y ciclos; sin embargo, se debe tener presente que existe un punto de saturación donde el tiempo de cómputo ya no es relevante para mejorar las soluciones. Las reconfiguraciones encontradas por el método aleatorio fueron apertura de las líneas: $31-32,60-62$, reconfigurar los circuitos 51 - 49 y 51 - 52 en el circuito 49 - 52 utilizando la configuración de la subestación 52; por último, se configuró la atención radial de la carga 66 a través del transformador $65-66$. Los tiempos de ejecución de la ILS para encontrar topologías que mejoran, por lo menos tres puntos la función objetivo respecto la del caso base, están entre ocho y cincuenta minutos. Esto implica que bajo una amplia combinación de paramentos, el método logra encontrar topologías con aumento de seguridad.

\section{Solución Utilizando SA}

EI SA aplicado se probó con 10 simulaciones combinando dos parámetros: la velocidad de enfriamiento y el número de repeticiones. Las temperaturas final e inicial se consideraron constantes con valores de 100 y 1 , respectivamente. En la Tabla 2 se muestran los resultados para la combinación de los parámetros mencionados. Se resalta que a través de este método se encuentra la topología que ofrece un mayor aumento de seguridad y que los tiempos de ejecución son muy bajos respecto a la cantidad de simulaciones que emplea la ILS. Las reconfiguraciones encontradas por el método SA fueron la apertura de las líneas: 31 - 32, 44 - 45, 45 - 49, 46 - 47, 54 - 59 y la atención de la carga 54 de manera radial desde el 
transformador 54 - 126. El método encontró soluciones con valor de función objetivo entre 57 y 65 , lo que implica que hubo búsquedas que no arrojaron topologías que mejoran la seguridad, aunque también se encontró la solución con mejor valor de función objetivo para el SEP probado.

Tabla 1: Resumen de resultados para ILS.

\begin{tabular}{|c|c|c|c|c|}
\hline $\begin{array}{c}\text { Número de } \\
\text { iteraciones }\end{array}$ & Distancia & Ciclos & Función objetivo & Tiempo hh:mm:ss \\
\hline 50 & 1 & 2 & 60 & $0: 44: 07$ \\
\hline 50 & 1 & 5 & 60 & $0: 52: 54$ \\
\hline 50 & 2 & 2 & 60 & $0: 38: 31$ \\
\hline 50 & 2 & 5 & 61 & $0: 42: 05$ \\
\hline 40 & 1 & 2 & 58 & $0: 31: 18$ \\
\hline 40 & 1 & 5 & 61 & $0: 35: 50$ \\
\hline 40 & 2 & 2 & 60 & $0: 30: 14$ \\
\hline 40 & 2 & 5 & 62 & $0: 34: 55$ \\
\hline 30 & 1 & 2 & 61 & $0: 20: 58$ \\
\hline 30 & 1 & 5 & 62 & $0: 23: 36$ \\
\hline 30 & 2 & 2 & 61 & $0: 22: 14$ \\
\hline 30 & 2 & 5 & 60 & $0: 23: 30$ \\
\hline 20 & 1 & 2 & 61 & $0: 14: 09$ \\
\hline 20 & 1 & 5 & 62 & $0: 15: 32$ \\
\hline 20 & 2 & 2 & 63 & $0: 15: 02$ \\
\hline 20 & 2 & 5 & 60 & $0: 16: 15$ \\
\hline 10 & 1 & 2 & 65 & $0: 07: 36$ \\
\hline 10 & 1 & 5 & 65 & $0: 08: 37$ \\
\hline 10 & 2 & 2 & 60 & $0: 07: 31$ \\
\hline 10 & 2 & 5 & 62 & $0: 08: 37$ \\
\hline
\end{tabular}

Tabla 2: Resumen de resultados para el Recocido Simulado.

\begin{tabular}{|l|l|l|l|}
\hline $\begin{array}{l}\text { Velocidad de } \\
\text { enfriamiento }\end{array}$ & Repeticiones & Función objetivo & Tiempo hh:mm:ss \\
\hline 1 & 2 & 62 & $0: 07: 59$ \\
\hline 1 & 5 & 57 & $0: 21: 03$ \\
\hline 2 & 2 & 62 & $0: 04: 22$ \\
\hline 2 & 5 & 62 & $0: 10: 42$ \\
\hline 3 & 2 & 62 & $0: 03: 05$ \\
\hline 3 & 5 & 62 & $0: 07: 55$ \\
\hline 4 & 2 & 65 & $0: 02: 25$ \\
\hline 4 & 5 & 62 & $0: 05: 46$ \\
\hline 5 & 2 & 64 & $0: 01: 55$ \\
\hline 5 & 5 & 61 & $0: 04: 41$ \\
\hline
\end{tabular}

\section{Simulaciones con diferentes tipos de subestación de manera no simultánea}

Para comparar las bondades de los algoritmos implementados se ejecutó otro conjunto de simulaciones sobre el SEP de prueba. Considerando las subestaciones 23, 47, 32, 42, 66, 45, 62, 51, 49, 69, 54, 50, 48 se realizó la transformación de las mismas a tipo interruptor y medio; luego, se ejecutó la búsqueda topológica utilizando los métodos implementados. Posteriormente, las mismas subestaciones fueron convertidas a tipo doble barra y anillo para ejecutar el proceso de búsqueda con los algoritmos desarrollados. En la Fig. 6 se presentan los resultados de las simulaciones realizadas. Los resultados se han agrupado por método de búsqueda y tipos de subestación consideradas. Las siglas utilizadas para hacer referencia a los tipos de subestación son: IM para interruptor y medio, DB para doble barra y AN para anillo.

El número ubicado en la parte superior de las barras indica la disminución de violaciones respecto el caso sin reconfiguraciones al realizar el análisis $\mathrm{N}-1$. Por ejemplo, en las primeras dos barras de la izquierda se puede interpretar que para subestaciones tipo IM, al aplicar SA se obtienen 57 violaciones de seguridad (8 menos que en el caso base) y al aplicar ILS se obtienen 58 violaciones de seguridad ( 7 menos que en el caso base). De forma análoga se interpretan las barras para los tipos de subestación DB y AN. Los resultados muestran que el SEP de pruebas con subestaciones tipo IM, al tener más alternativas de reconfiguración que las $\mathrm{DB}$ y $\mathrm{AN}$, obtiene mayores niveles de seguridad al ser reconfigurada. Por otra parte, 
cuando se utilizaron subestaciones tipo AN se obtuvo el menor incremento de seguridad. Se observa que el mayor incremento de condiciones de seguridad fue de ocho con el método SA en el SEP con subsecciones tipo IM.

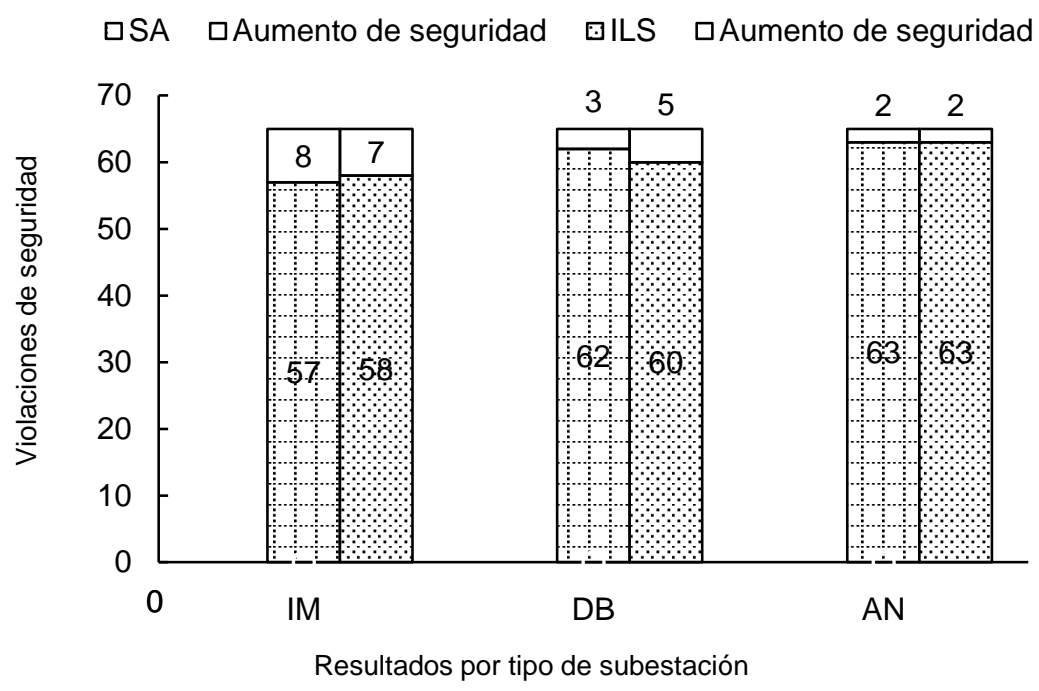

Fig. 6: Resultados agrupados, por tipo de subestación, de las condiciones de seguridad alcanzadas con los diferentes métodos de búsqueda.

Con el objetivo de mostrar la capacidad del método de encontrar soluciones compuestas por diferentes tipos de subestaciones y su aplicación en porciones de un SEP (buscando el acotamiento del problema de acuerdo al interés del usuario), se realizó una división del SEP de pruebas por zonas de acuerdo a la Tabla . Las subestaciones que harán parte de la solución inicial fueron seleccionadas de acuerdo al número de bahías que las conforman. En cada zona se seleccionaron las doce subestaciones con el mayor número de bahías. Para combinar la solución con subestaciones de diferentes tipos, se planteó que las cuatro subestaciones más grandes fueran tipo IM, debido a que otorgan mayor flexibilidad. Considerando el criterio del mayor número de bahías se asignaron las subestaciones tipo DB y AN, respectivamente. En la Tabla 3 se presenta las barras que componen cada una de las soluciones iniciales y el tipo de subestaciones que se utilizó en la búsqueda.

La metodología acotada por zonas consiste en solo realizar el análisis N-1 de los elementos que componen la misma y realizar cambios topológicos solo en el área acotada. Sin embargo, el monitoreo de las violaciones por sobrecarga y tensiones se realiza en todo el sistema. Esto con el fin de poder cuantificar el impacto de los cambios topológicos de una zona en las demás. Con lo anterior, se busca focalizar la búsqueda topológica en solucionar problemas de sobrecarga en partes específicas de la red.

Tabla 3: Subestaciones candidatas para la búsqueda topológica clasificadas por configuración y zona.

\begin{tabular}{|l|l|l|l|l|l|l|l|l|l|l|l|l|}
\hline Zona & \multicolumn{3}{|c|}{ Interruptor y medio } & \multicolumn{4}{c|}{ Doble barra } & \multicolumn{3}{c|}{ Anillo } \\
\hline 1 & B23 & B31 & B11 & B24 & B32 & B19 & B27 & B5 & B15 & B70 & B17 & B12 \\
\hline 2 & B37 & B66 & B34 & B69 & B105 & B56 & B59 & B54 & B80 & B100 & B49 & B77 \\
\hline 3 & B86 & B91 & B102 & B93 & B103 & B90 & B83 & B101 & B85 & B89 & B110 & B92 \\
\hline
\end{tabular}

Las simulaciones se realizaron con todos los métodos de búsqueda implementados y sobre todas las zonas descritas anteriormente. Los resultados de las simulaciones son presentados en la Fig. 7, donde cada grupo de tres columnas representa una zona, y las barras muestran los resultados en términos del número de violaciones después de realizar las modificaciones topológicas encontradas por cada uno de los algoritmos de búsqueda. En la Fig. 7, la suma de los números que componen cada barra muestran el número de violaciones de seguridad antes de aplicar las modificaciones topológicas. Los números sobre los rectángulos presentan la disminución de violaciones causadas por las reconfiguraciones. Se puede observar que en la Zona 1 se disminuyeron hasta 11 condiciones de inseguridad realizando cambios de topología. Por su parte, en la Zona 2 fueron ocho, mientras que en la Zona 3, solo una. La razón principal que permite encontrar el mejor valor de función objetivo en la Zona 1 es su tamaño y las características de las subestaciones que componen la zona, pues estas tienen una mayor conectividad que las subestaciones de las demás zonas. 


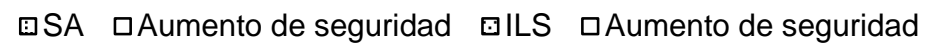

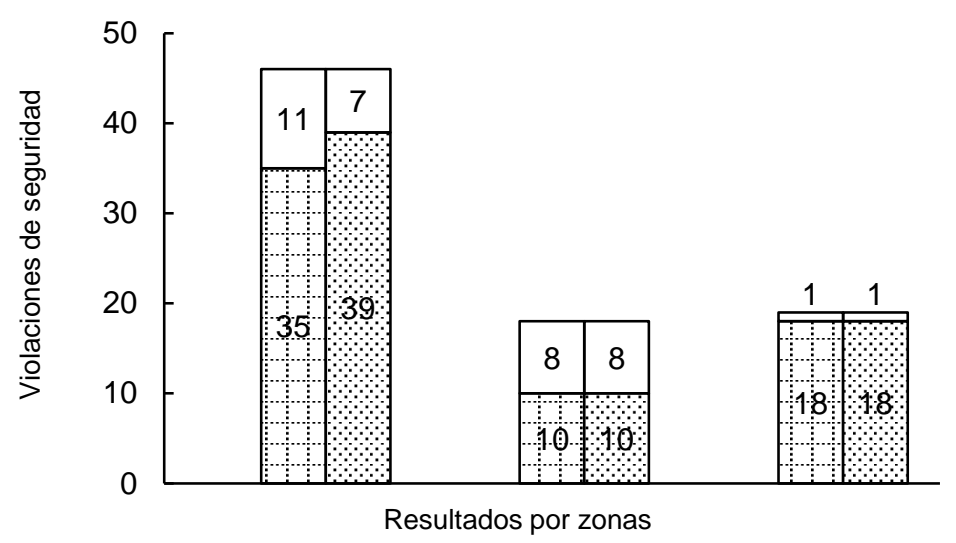

Fig. 7: Resultados agrupados por tipo de subestación de las condiciones de seguridad alcanzadas con los diferentes métodos de búsqueda aplicados.

\section{CONCLUSIONES}

En este artículo se presentaron dos técnicas para aumento de seguridad en sistemas de potencia mediante modificaciones topológicas. Los resultados con el sistema IEEE de 118 barras evidenciaron la capacidad de los algoritmos de aumentar la seguridad del sistema mediante la reconfiguración de subestaciones tipo interruptor y medio, doble barra y anillo. Las técnicas propuestas pueden aplicarse en un sistema completo o en una región acotada del mismo.

La topología bahía - interruptor utilizada en este trabajo permite evaluar de forma más realista los impactos de las contingencias $\mathrm{N}-1$. Cuando se modela esta topología, para una contingencia dada dos elementos adicionales del SEP deben ser abiertos. La consideración del modelo bahía - interruptor es un factor diferenciador con respecto a otros trabajos reportados en la literatura técnica; pues las soluciones entregadas por modelos que no consideren este tipo de topología deben ser validadas en una etapa posterior al proceso de búsqueda para verificar los impactos reales de las modificaciones topológicas.

A partir de las simulaciones realizadas, se logró determinar que los métodos de búsqueda implementados pueden ser utilizados en SEP de cualquier tamaño. Su capacidad de trabajar en regiones acotadas de un SEP le brinda el potencial necesario para intervenir las áreas operativas que el usuario de la herramienta determine. El acotamiento de la red es importante para evitar exploraciones de topologías que no tienen influencia sobre problemas de red detectados.

En cuanto al desempeño de las metaheurísticas implementadas se pudo observar que respecto al tiempo de ejecución el SA pudo encontrar soluciones más rápido que la ILS. Por otro lado, el SA también encontró soluciones de mejor calidad. Ambas metaheurísticas dependen de un número similar de parámetros que deben ser calibrados al inicio del algoritmo. En este sentido, el desempeño de una u otra metaheurística depende del tipo de sistema bajo estudio y su parametrización; por lo tanto, no se puede afirmar con certeza cual de las dos técnicas es mejor.

\section{NOMENCLATURA}

\section{Variables}

$O L_{i, l}$ : Variable binaria que toma el valor de uno si la contingencia $i$ produce una sobrecarga en la rama Iy cero en caso contrario.

$O V_{i, n}$ : Variable binaria que toma el valor de uno si la contingencia $i$ produce una tensión por fuera de los límites en la barra $n$.

$C V_{r}$ : Variable binaria que toma el valor de uno cuando no hay convergencia ante la contingencia $r \mathrm{y}$ cero en caso contrario.

$D X_{s}: \quad$ Variable binaria que toma el valor de uno cuando una carga o generador $s$ es desconectado y cero en caso contrario.

$P_{D S_{n}}: \quad$ Demanda no atendida en el nodo $n$.

$P_{n}: \quad$ Potencia activa neta inyectada en el nodo $n$.

$Q_{n}$ : $\quad$ Potencia reactiva neta inyectada en el nodo $n$.

$P_{l}^{B r}: \quad$ Flujo de potencia activa en la rama $I$. 
$Q_{l}^{B r}: \quad$ Flujo de potencia reactiva en la rama $I$.

$S_{l}^{B r}: \quad$ Flujo de potencia aparente en la rama $l$.

T: $\quad$ Temperatura.

fo: $\quad$ Función objetivo.

\section{Parámetros}

$k_{1}, k_{2}: \quad$ Constantes de penalización de la función objetivo

$P_{D_{n}}: \quad$ Demanda del nodo $n$.

$G_{m n}$ : $\quad$ Componente real de la position $m, n$ de la matriz de admitancia nodal del sistema.

$B_{m n}$ : Componente imaginario de la position $m, n$ de la matriz de admitancia nodal del sistema.

$V_{n}: \quad$ Magnitud de la tensión en el nodo $n$.

$\theta_{m n}: \quad$ Apertura angular entre las barras $m, n$.

$g_{m n}: \quad$ Conductancia de la rama que conecta los nodos $m, n$.

$b_{m n}: \quad$ Susceptancia de la rama que conecta los nodos $m, n$.

$T_{o}$ : $\quad$ Temperatura inicial del recocido simulado.

Tfin: Temperatura final del recocido simulado.

nrep: $\quad$ Número de iteraciones.

$d t: \quad$ Constante de reducción de temperatura para el recocido simulado.

$\beta$ : $\quad$ Diferencia entre el valor de dos funciones objetivo consecutivas para el recocido simulado.

$\theta_{\text {ref }}: \quad$ Ángulo de referencia.

$P_{g}^{G e n}: \quad$ Generación de potencia activa.

$Q_{g}^{G e n}: \quad$ Generación de potencia reactiva.

\section{Conjuntos}

Ce: $\quad$ Conjunto de contingencias

$B r: \quad$ Conjunto de ramas

$N$ : $\quad$ Conjunto de nodos

$N C e: \quad$ Conjunto de contingencias para las que no hay convergencia del flujo de carga

Dcg: $\quad$ Conjunto de cargas y generadores desconectados debido a una contingencia

\section{REFERENCIAS}

Bai, Y., H. Zhong, Q. Xia y C. Kang, A Two-Level Approach to AC Optimal Transmission Switching with an Accelerating Technique, DOI: 10.1109/TPWRS.2016.2582214, IEEE Transactions on Power Systems, 32(2), 1616-1625 (2016)

Fuller, J.D., R. Ramasra y A. Cha, Fast Heuristics for Transmission-Line Switching, DOI: 10.1109/TPWRS.2012.2186155, IEEE Transactions on Power Systems, 27(3), 1377-1386 (2012)

Granelli, G.P., M. Montagna, F. Zanellini, P. Bresesti y R. Vailati, A genetic algorithm-based procedure to optimize system topology against parallel flows, DOI: 10.1109/TPWRS.2005.860921, IEEE Transactions on Power Systems, 21(1), 333-340 (2006)

Heidarifar, M. y H. Ghasemi, A Network Topology Optimization Model Based on Substation and Node-Breaker Modeling, DOI: 10.1109/TPWRS.2015.2399473, IEEE Transactions on Power Systems, 31(1), 247-255 (2016)

Jiajun L.V., T. Ding, Z. Bie y X. Wang, A Novel Linearization Variant of Reliability Costs in the Optimal Scheduling Model, DOI: 10.1109/TPWRS.2017.2650783, IEEE Transactions on Power Systems, 32(5), 4140-4142 (2017)

Khanabadi, M., H. Ghasemi y M. Doostizadeh, Optimal Transmission Switching Considering Voltage Security and N-1 Contingency Analysis, DOI: 10.1109/TPWRS.2012.2207464, IEEE Transactions on Power Systems, 28(1), 542-550 (2013)

Korad, A. S. y K. W. Hedman, Robust Corrective Topology Control for System Reliability, DOI: 10.1109/TPWRS.2013.2267751, IEEE Transactions on Power Systems, 28(4), 4042-4045 (2013)

López, J. M., J. Cortina y N. Muñoz, Assessment of the Electric Grid Interdiction Problem using a Nonlinear Modeling approach, DOI: 10.1016/j.epsr.2016.12.017, Electric Power Systems Research, 144(1), 243-254 (2017)

López, J.M., F. Villada y N. Muñoz, Metaheurísticas Aplicadas a la Ubicación y Dimensionamiento Óptimo de Generación Distribuida en Sistemas de Distribución de Energía Eléctrica, DOI: 10.4067/S0718-07642015000400018, Revista Información Tecnológica, 26(4), 145-158 (2015)

Mazi, A.A., B. F. Wollenberg y M. H. Hesse, Corrective Control of Power System Flows by Line and Bus-bar Switching, DOI: 10.1109/TPWRS.1986.4334990, IEEE Transactions on Power Systems, 1(3), 258-264 (1986) 
Parvini, Z., A. Abbaaspour, M. Fotuhi-Firuzabad y M. Moeini-Aghtaie, Operational Reliability Studies of Power Systems in the Presence of Storage Systems, DOI: 10.1109/TPWRS.2017.2771521, IEEE Transactions on Power Systems, 33(4), 3691-3700 (2018)

Quintero, C.M., Aumento de la Seguridad en Sistemas de Potencia en Condiciones no Convencionales de Operación Mediante Modificaciones Topológicas de la Red, Universidad de Antioquia (2018)

Rodriguez, A.B., F. Saavedra y L.J. Ramírez, Estrategia Metaheurística para Redes Ópticas sin Conversión de Longitud de Onda con Tráfico Dinámico (WDM), DOI: 10.4067/S0718-07642016000500023, Revista Información Tecnológica, 27(5), 221-230 (2016)

Saldarriaga-Zuluaga, S.D, López-Lezama, J.M. y Muñoz-Galeano, N., Planeamiento Integrado de la Expansión en Generación y Transmisión Considerando Restricciones de Seguridad, DOI: 0.4067/S0718-07642018000300167, Revista Información Tecnológica, 29(3), 167-176 (2018)

Shao, W. y V. Vittal, Corrective switching Algorithm for Relieving Overloads and Voltage Violations, DOI: 10.1109/TPWRS.2005.857931, IEEE Transactions on Power Systems, 20(4), 1877-1885 (2005)

Soroush, M. y J.D. Fuller, Accuracies of Optimal Transmission Switching Heuristics Based on DCOPF and ACOPF, DOI: 10.1109/TPWRS.2013.2283542, IEEE Transactions on Power Systems, 29(2), 924-932 (2014)

Yang, H., L. Wang, Y. Zhang, H.M. Tai, Y. Ma y M. Zhou, Reliability Evaluation of Power Systems Considering Time of Use Electricity Pricing, DOI: 10.1109/TPWRS.2018.2879953, IEEE Transactions on Power Systems, 34(3), 1991-2022 (2019)

Zhang, C. y J. Wang, Optimal Transmission Switching Considering Probabilistic Reliability, DOI: 10.1109/TPWRS.2013.2287999, IEEE Transactions on Power Systems, 29(2), 974-975 (2014) 
